\title{
La copia privada en España y los sistemas de compensación equitativa: estudio comparativo
}

The private copy in Spain and fair compensation systems: comparative study

\author{
José Luis GonZÁLEZ SAN JUAN \\ Abogado. C/ del Cáliz 15, 37001 Salamanca, España, \\ gonzalezsanjuan@icasal.com
}

\begin{abstract}
Resumen
Se realiza un estado de la cuestión sobre la compensación equitativa por copia privada en España. Después de delimitar brevemente el concepto de copia privada en el contexto de los derechos de autor, y de describir los antecedentes de la compensación equitativa en España, se analiza su régimen jurídico actual, comentando sus principales ventajas e inconvenientes. También se revisa la recaudación por copia privada en la última década y las previsiones sobre su evolución en los próximos años.
\end{abstract}

Palabras clave: Copia privada. Canon digital. Derechos de autor. Compensación equitativa. España.

\section{Introducción}

Los derechos de autor, cuyo objeto son principalmente las obras artísticas y literarias, surgieron en los siglos XVIII y XIX para incentivar la creación y la inversión en bienes culturales, con el convencimiento de que redundarían en beneficio de toda la sociedad (Carbajo, 2010, p. 128).

Se reconocieron unos derechos exclusivos y excluyentes, a los autores sobre sus obras, para que, creando una escasez artificial en el mercado, se les facilitara la obtención de una compensación económica por el uso y disfrute que de las mismas realizaran terceros.

$\mathrm{Y}$, para mantener el necesario y justo equilibrio entre estos nuevos derechos y el derecho de acceso a la cultura (Abbud y Fadel, 2010, p. 163), se establecieron límites y excepciones, que permitían algunos usos de las obras sin autorización, cumpliendo ciertas condiciones (González, 2017, p. 36).

Pero este frágil equilibrio se ha roto con la irrupción de las Tecnologías de la Información y las Comunicaciones (TIC), que facilitan enormemente la realización de copias de alta calidad de las obras (Riera, 2001, p.119), especialmente canciones, libros y películas (1), y también su transmisión en tiempo real, provocando un incremento sin precedentes de la piratería de

\begin{abstract}
A state of the question on compensation for private copying in Spain is outlined. We first briefly describe the concept of private copying in the context of copyright, and the antecedents of fair compensation in Spain. Them its current legal regime is analyzed, commenting its main advantages and disadvantages. We also review the collection by private copy in the last decade and the forecasts on its evolution in the coming years.
\end{abstract}

Keywords: Private copy. Digital canon. Copyright. Fair compensation. Spain.

contenidos, tanto doméstica como profesional (Carbajo, 2002, p. 21).

Así, en esta nueva era tecnológica, la compensación equitativa por copia privada es esencial para recuperar el equilibrio perdido, y por ello, cualquier cambio en su regulación suscita controversias entre todos los actores implicados.

\section{Objetivo y metodología}

El objetivo del presente estudio es revisar, desde un punto de vista crítico, los diferentes sistemas de compensación equitativa por copia privada que se han sucedido en el ordenamiento jurídico español, así como analizar las recaudaciones obtenidas hasta la fecha, y su previsible evolución en el futuro próximo.

Para ello, hemos utilizado una metodología de tipo mixto, partiendo de una búsqueda de datos y bibliografía, tanto en relación con las reformas legislativas como con las cifras de recaudación, para después ordenar y analizar dichos datos.

Como fuentes principales en lo que respecta a las cifras de recaudación, se han utilizado las publicaciones oficiales de la Organización Mundial de la Propiedad Intelectual (OMPI), así como las memorias anuales de dos de las más conocidas sociedades de gestión de derechos: la Asociación de Autores y Editores de Libros, Revistas, 
Periódicos y Partituras (CEDRO) y la Sociedad General de Autores y Editores (SGAE).

\section{El límite de copia privada}

\subsection{Normativa aplicable}

Las tres principales normas a considerar en España, en relación con el límite de copia privada, son: a) Directiva 2001/29 CE de Derechos de Autor, en adelante DDA (DOUE, 2001); b) Texto Refundido de la Ley de Propiedad Intelectual (BOE, 1996), en adelante TRLPI; c) Real Decreto-ley 12/2017, que modifica el TRLPI en relación con la compensación por copia privada (BOE, 2017), en adelante RD-L 12/2017.

\subsection{Concepto de copia privada}

La copia privada es un límite al derecho de reproducción que permite a un tercero, sin autorización del titular de los derechos, realizar copias de obras protegidas ya divulgadas, siempre que se utilicen exclusivamente en un ámbito privado y sin fines comerciales directos o indirectos.

Por ello, no existe un derecho subjetivo de los usuarios a la copia privada, pues en puridad, se trata de una limitación del derecho de reproducción de los autores (Aparicio, 2016, p. 27).

El requisito de que la copia realizada se utilice en el ámbito privado, impide su aplicación en casos donde se exceda dicho ámbito, por ejemplo, compartir la obra en una red P2P (peer to peer) o en una Web de enlaces (Carbajo, 2010, p. 129; Aparicio, 2016, p. 31).

En la mayoría de los ordenamientos jurídicos, incluido el español, la copia privada solo alcanza a las personas físicas, quedando excluidas las jurídicas, y también suele exigirse que la reproducción se realice a partir de una fuente lícita y sin vulnerar las condiciones de acceso a la obra o prestación (art. 31.2 TRLPI).

\subsection{Justificación del límite de copia privada}

La razón de ser del límite de copia privada es doble, pues por una parte se fundamenta en razones de interés social, relacionadas con la facilitación de la difusión y el acceso a la cultura, y por otra, en poderosos motivos de índole prácticos, al resultar imposible para los autores controlar este tipo de copias, o al menos, hacerlo de una forma que resulte económicamente viable (Aparicio, 2016, p. 27).

\subsection{Alcance y exclusiones}

En España, la copia privada comprende tres tipos de obras: a) libros y publicaciones asimiladas; b) fonogramas y otros soportes sonoros; c) videogramas y otros soportes visuales o audiovisuales (art. 25.1 TRLPI).

Quedan excluidos expresamente los programas de ordenador y las bases de datos, a tenor de los artículos 31.3.b y 31.3.c TRLPI, respectivamente. No obstante, en los programas de ordenador se permite realizar copias de seguridad, concepto mucho más limitado que el de la copia privada, pero esta previsión no existe para las bases de datos (Aparicio, 2016, p. 32).

También quedan excluidas las obras puestas a disposición del público (normalmente en Internet), en las que se autoriza la reproducción gratuita u onerosa, conforme a lo convenido por contrato (art. 31.3.a TRLPI).

\subsection{Compensación equitativa}

En la mayoría de los ordenamientos jurídicos, el límite de copia privada está asociado a una compensación equitativa, recaudada y repartida normalmente por asociaciones de los titulares de los derechos. En el ámbito de la UE esta compensación es obligatoria, a tenor del art. 5.2.b de la DDA, pero siempre que exista el límite de copia privada, puesto que éste es potestativo.

En España, la compensación equitativa por copia privada ha generado una fuerte polémica desde su implantación, sucediéndose diversos cambios legislativos provocados por las sentencias del Tribunal de Justicia de la UE (TJUE), como veremos.

Por ello, puede defenderse la existencia de responsabilidad patrimonial del Estado, ya que con su actuación u omisión ha privado a los autores de una parte de la compensación equitativa a la que tenían derecho (García, 2017, p.11; Carbajo, 2016, p. 16).

\subsection{Medidas Tecnológicas de Protección (MTP)}

Las MTP son cualquier técnica, dispositivo o componente que, en su funcionamiento normal, esté destinado a impedir o restringir vulneraciones de los derechos de autor (art. 160.3 TRLPI).

Los sistemas de gestión de derechos digitales o DRM (Digital Rights Management), son también MTP, existiendo entre ambas figuras una relación género/especie, siendo las MTP el género y los DRM la especie (González, 2016, p 72).

Las MTP son una herramienta poderosa que las TIC ofrecen a los titulares de derechos para su defensa, pero el problema surge cuando éstas impiden o limitan la copia de las obras, pues entonces los usuarios no podrán realizar copias privadas, o verán limitada dicha posibilidad, no 
pareciendo lógico que dicha obra reciba la compensación equitativa (Riera, 2001, p. 122).

No obstante, el art. 161 TRLPI obliga a los titulares de derechos que aplican MTP, a facilitar a los beneficiarios de los límites su disfrute, y adicionalmente, el TJUE ha indicado que la existencia de MTP no supone eliminar la compensación equitativa, aunque sí que deberá tenerse en cuenta para determinar su cuantía (TJUE, 2015, \#71-73).

\section{Evolución de la compensación por copia privada en España}

Desde su implantación en España, la compensación equitativa por copia privada ha pasado por tres fases diferentes: inicialmente se implantó un sistema basado en canon sobre los soportes y aparatos utilizados para realizar las copias privadas (antiguo canon digital), posteriormente se pasó a uno con cargo a los Presupuestos Generales del Estado (PGE), y finalmente se retornó al sistema de canon (nuevo canon digital), que es el vigente en la actualidad.

\subsection{Antiguo canon digital}

La compensación equitativa por copia privada fue establecida en España por la ley 22/1987 (BOE, 1987), e incorporada posteriormente al TRLPI, pero los autores no percibieron cantidad alguna hasta el año 2003, cuando un acuerdo entre las entidades gestoras y ASIMELEC (Asociación Multisectorial de Empresas de Tecnologías de la Información, Comunicaciones y Electrónica), permitió su cobro (García, 2017, p. 2).

Este acuerdo se incluyó en la disposición transitoria única de la ley 23/2006 (BOE, 2006), fue desarrollado reglamentariamente por la Orden PRE/1743/2008 (BOE, 2008), y consistía en una compensación mediante canon sobre los equipos y soportes utilizados para la copia privada.

Pero, en el seno de una controversia judicial entre la SGAE y PADAWAN S.L. (empresa comercializadora de productos sujetos al canon), el TJUE determinó, en sentencia de octubre de 2010 (TJUE, 2010), que:

[...], la aplicación indiscriminada del canon por copia privada, en particular en relación con equipos, aparatos y soportes de reproducción digital que no se hayan puesto a disposición de usuarios privados y que estén manifiestamente reservados a usos distintos a la realización de copias privadas, no resulta conforme con la Directiva 2001/29.

Ya que las personas físicas son las únicas que pueden realizar copias privadas, y por ello el canon no puede aplicarse a equipos y soportes que no sean puestos a disposición de éstas.
Tras esta sentencia el legislador, en lugar de reformar el sistema de compensación basado en canon para adaptarlo a las resoluciones del TJUE, decidió modificarlo completamente, implantando un sistema con cargo a los PGE.

\subsection{Compensación con cargo a los PGE}

EI RD-L 20/2011 (BOE, 2011) y su reglamento de desarrollo, RD 1657/2012 (BOE, 2012), sustituyeron el sistema de compensación basada en canon por uno con cargo a los PGE, estableciendo una compensación global fija, a determinar anualmente en los PGE, en función del perjuicio causado a los titulares de derechos.

Adicionalmente, se limitó el alcance de la copia privada, con el claro objetivo de que las cifras obtenidas al calcular dicho perjuicio fueran sensiblemente menores.

Por ello, las asociaciones de gestión de derechos se opusieron frontalmente a esta modificación, ya que les provocaba una importante merma de ingresos, e iniciaron un procedimiento contencioso que derivó en una nueva cuestión prejudicial, elevada por el TS ante el TJUE.

En junio de 2016, el TJUE determinó que el sistema de compensación con cargo a los PGE era contrario al Derecho de la UE, al no garantizar que la compensación fuera soportada exclusivamente por los usuarios de copias privadas, que como ya hemos indicado, solo pueden ser las personas físicas (TJUE, 2016), y por ello, el TS anuló el RD 1657/2012 (BOE, 2012), en su sentencia de 10 de noviembre de 2016.

\subsection{Situación actual: nuevo canon digital}

El vacío legal provocado por los acontecimientos anteriores se prolongó durante muchos meses, hasta que en julio de 2017 se aprobó el RD-L 12/2017 (BOE, 2017), que reimplantó el sistema de compensación basado en canon sobre equipos, aparatos y soportes, aunque con una nueva regulación que lo adaptaba a la normativa de la UE.

En este nuevo sistema, los deudores indirectos de la compensación son los fabricantes y distribuidores de los productos, pudiendo repercutirse el canon a los usuarios finales, que son los deudores directos.

Los acreedores son los autores, artistas y ejecutantes, de forma irrenunciable, así como también los editores y productores, a los que sí se les permite la renuncia.

EI RD-L 12/2017 establece que la lista de productos afectados por el canon se fijará, en el plazo máximo de un año desde su entrada en vigor, 
mediante reglamento de desarrollo, aplicándose hasta entonces una lista provisional incluida en su disposición transitoria segunda.

Para adecuarse a las resoluciones del TJUE se introducen, en el art. 25.7 TRLPI, varias exceptuaciones (sector público, personas jurídicas consumidores finales con uso exclusivamente profesional, etc.).

Adicionalmente, las personas físicas o jurídicas no exceptuadas del pago, podrán solicitar el reembolso del canon si los productos se destinan a la exportación o entrega intracomunitaria, y también cuando sean consumidores finales y justifiquen el destino exclusivamente profesional de los mismos.

\section{Gestión del canon y forma de reparto}

Para la gestión del cobro y reparto de la recaudación, así como para la tramitación de las exceptuaciones y reembolsos, se estableció que las sociedades de gestión debían crear una nueva persona jurídica (art. 25.10 TRLPI).

Esta persona jurídica, que fue creada en 2017, se denomina Ventanilla Única Digital (en adelante VUD), y es una asociación formada por las ocho entidades de gestión de derechos a las que les afecta la copia privada: AGEDI (Asociación de Gestión de Derechos Intelectuales), DAMA (Derechos de Autor de Medios Audiovisuales), AIE (Sociedad de Artistas, Intérpretes o Ejecutantes), VEGAP (Visual Entidad de Gestión de Artistas Plásticos), AISGE (Artistas Intérpretes, Sociedad de Gestión), EGEDA (Entidad de Gestión de Derechos de los Productores Audiovisuales), SGAE y CEDRO.

\begin{tabular}{|c|c|c|c|}
\hline & Libros & Audio & Video \\
\hline CEDRO & $87,50 \%$ & & \\
\hline VEGAP & $12,50 \%$ & & $1,333333 \%$ \\
\hline EGEDA & & & $33,333333 \%$ \\
\hline AISGE & & & $26,666667 \%$ \\
\hline DAMA & & & $2,553333 \%$ \\
\hline SGAE & & $50,00 \%$ & $29,446667 \%$ \\
\hline AIE & & $25,00 \%$ & $6,666667 \%$ \\
\hline AGEDI & & $25,00 \%$ & \\
\hline
\end{tabular}

Tabla I. Reparto entre entidades de gestión (http://ventanillaunica.digital/):

Ante la falta de regulación al respecto, estas entidades han llegado a un acuerdo privado, para fijar las cantidades que, en cada equipo o soporte, irán destinadas a cada modalidad de reproducción (libros, audio y video), y para establecer los porcentajes a asignar a cada entidad en cada modalidad de reproducción, que son los que se muestran en la tabla I. Posteriormente, cada entidad de gestión distribuirá las cantidades que le hayan correspondido, entre los diferentes actores que intervienen en el proceso de creación y producción de las obras, de acuerdo a su normativa interna, y reservando un porcentaje para fines asistenciales, formación y promoción de sus socios.

Por ejemplo CEDRO, tras reservar el $20 \%$ para fines asistenciales, formación y promoción, reparte la recaudación por copia privada entre los autores y editores de los libros y publicaciones asimiladas, que hayan sido publicados en los tres años anteriores al del cobro, asignando el $55 \%$ a los autores y el $45 \%$ a los editores, y teniendo en cuenta además otros factores, como el precio de venta de las publicaciones y su temática.

\section{Evolución de la recaudación por copia privada en España}

En la tabla II pueden verse las cantidades devengadas en España por copia privada en la última década, y la previsión para el año 2018.

\begin{tabular}{rrr}
\hline Año & $\begin{array}{r}\text { Cantidad } \\
\text { (miles } €)\end{array}$ & \multicolumn{2}{c}{$\begin{array}{c}\text { Variación } \\
\text { (\%) }\end{array}$} \\
\hline 2007 & 40.706 & 47,4 \\
\hline 2008 & 59.992 & 31,8 \\
\hline 2009 & 79.080 & 3,9 \\
\hline 2010 & 82.176 & $-24,9$ \\
\hline 2011 & 61.710 & $-91,9$ \\
\hline 2012 & 5.000 & 0,0 \\
\hline 2013 & 5.000 & 0,0 \\
\hline 2014 & 5.000 & $-100,0$ \\
\hline 2015 & 0 & 0,0 \\
\hline 2016 & 0 & (agosto-diciembre) \\
\hline 2017 & 28.847 & (estimación) \\
\hline 2018 & 70.000 & \\
\hline
\end{tabular}

Tabla II. Cantidades devengadas por copia privada en España

Los datos de los años 2007 al 2015 son los publicados por la OMPI (Wijminga et al., 2017, p. 16), en el 2016 no se devengó cantidad alguna, y los datos del 2017 provienen del informe ejecutivo de dicho año de la entidad Ventanilla Única Digital (VUD, 2017, p. 2). 
No obstante, en relación con el año 2017, es necesario realizar dos precisiones. En primer lugar, al entrar en vigor el RD-L 12/2017 el 1 de agosto, la cantidad devengada en el 2017 se corresponde exclusivamente con los últimos cinco meses del año.

$\mathrm{Y}$, en segundo lugar, debe tenerse en cuenta que aún no ha sido promulgado el reglamento de desarrollo, de forma que tanto la lista de productos afectados por el canon, como las cantidades asignadas a los mismos, pueden verse modificadas en el futuro.

Por otra parte, la cifra del 2018 es nuestra propia previsión, y ha sido obtenida extrapolando la recaudación de los cinco meses del año 2017 en que ha estado vigente el nuevo canon digital.

Esta cifra, que hemos estimado en unos 70 millones, es muy similar a los niveles devengados en los años anteriores al 2011 (antiguo canon digital), pero queda bastante lejos de algunas de las previsiones realizadas, que situaban esta recaudación en el intervalo de los 110 a los 125 millones de euros anuales (Sánchez, 2017).

Los datos anteriores los hemos representado gráficamente en la figura 1, donde se aprecia mucho mejor la espectacular caída de la recaudación sufrida tras implantarse el sistema con cargo a los PGE, al pasar ésta de los $64.733 €$ (que fue la media de los años 2007 a 2011), a los $5.000 €$ fijados en los PGE, lo que supone una reducción del $92,27 \%$ (y eso sin contar que con cargo a los años 2015 y 2016 no se ha devengado aún cantidad alguna).

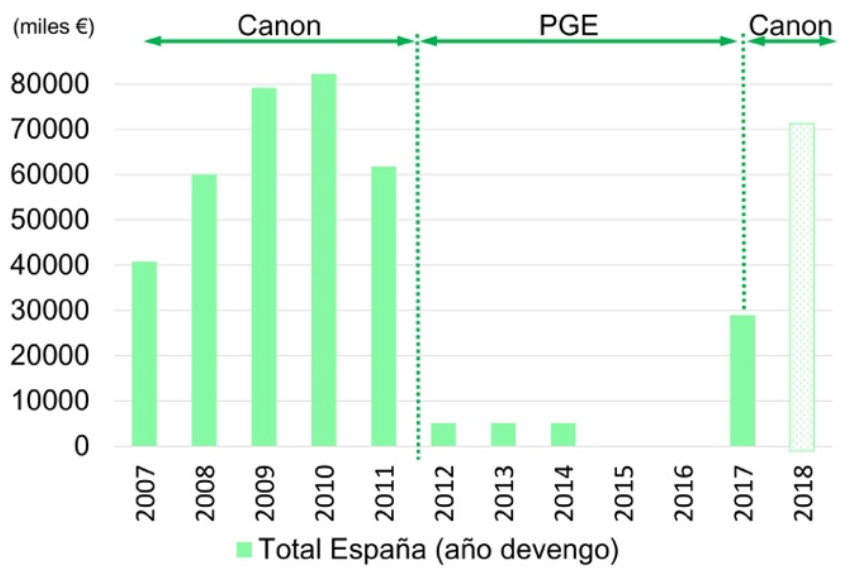

Figura 1. Representación de las cantidades totales devengadas en España por copia privada

En la figura anterior, también se pone claramente de manifiesto la no menos espectacular recuperación de la recaudación una vez implantado el nuevo canon digital, claramente observable ya en el año 2017, a pesar de que las cantidades devengadas en este año se corresponden únicamente con las de sus últimos cinco meses.

Así pues, la principal consecuencia de los cambios del sistema de compensación en España ha sido que las entidades de gestión de derechos, y por tanto también los titulares de éstos, sufrieron una importante merma en sus ingresos a partir del año 2011 y hasta el 2016, como puede apreciarse en la tabla III y en la figura 2, que reflejan las cantidades recaudadas por dos de estas sociedades, CEDRO y SGAE, aunque todo indica que volverán a recuperarlos con el sistema actual.

\begin{tabular}{lrrrr}
\hline \multirow{2}{*}{ Año } & \multicolumn{2}{c}{ CEDRO } & \multicolumn{2}{c}{$S G A E$} \\
\cline { 2 - 5 } & $\begin{array}{l}\text { Cantidad } \\
\text { (miles } € \text { ) }\end{array}$ & $\begin{array}{c}\text { Variación } \\
(\%)\end{array}$ & $\begin{array}{r}\text { Cantidad } \\
(\text { miles } €)\end{array}$ & $\begin{array}{c}\text { Variación } \\
(\%)\end{array}$ \\
\hline 2006 & 41.120 & - & 20.985 & - \\
\hline 2007 & 43.900 & 6,7 & 20.478 & $-2,4$ \\
\hline 2008 & 37.880 & $-13,7$ & 16.009 & $-21,8$ \\
\hline 2009 & 21.980 & $-41,9$ & 27.677 & 72,9 \\
\hline 2010 & 19.920 & $-9,3$ & 28.156 & 1,7 \\
\hline 2011 & 19.290 & $-3,1$ & 20.247 & $-28,1$ \\
\hline 2012 & 4.890 & $-74,6$ & 4.231 & $-79,1$ \\
\hline 2013 & 3.990 & $-18,4$ & 2.691 & $-36,4$ \\
\hline 2014 & 2.780 & $-30,3$ & 2.800 & 4,1 \\
\hline 2015 & 990 & $-64,3$ & 10.480 & 274,3 \\
\hline 2016 & 0 & $-100,0$ & 428 & -95.9 \\
\hline 2017 & 1.654 & - & - & - \\
\hline 2018 & 10.000 & (estimación) & - & - \\
\hline
\end{tabular}

Tabla III. Evolución de la recaudación por copia privada de CEDRO y $S G A E$

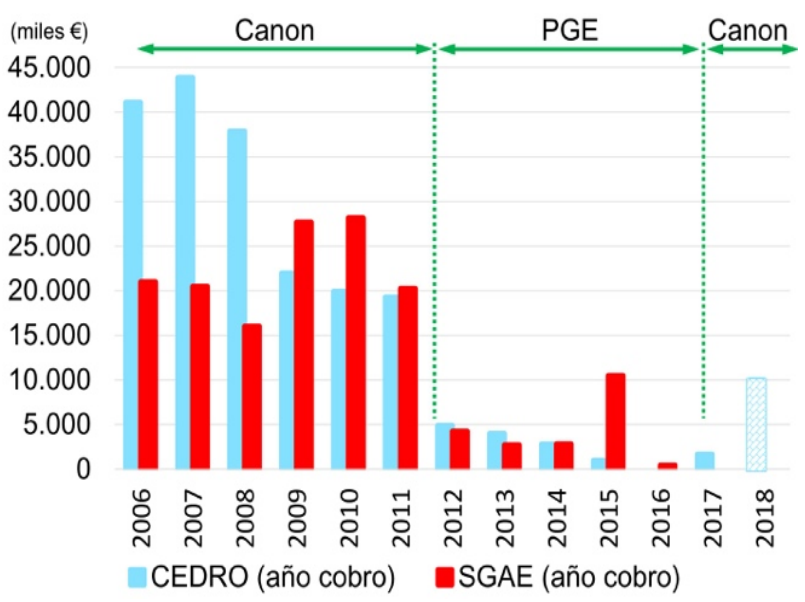

Figura 2. Representación gráfica de la recaudación por copia privada de CEDRO y SGAE 
Los datos anteriores se han obtenido de las memorias anuales de dichas sociedades (CEDRO, 2018; SGAE, 2018), pero debe tenerse en cuenta que no se corresponden con las cantidades devengadas en cada ejercicio, pues los ingresos se producen normalmente con varios meses de retraso, y también se incluyen ciertos ajustes, como por ejemplo cantidades recaudadas por acuerdos puntuales, reclamaciones judiciales o meros atrasos (2).

En cuanto a las cifras del 2018, éstas se han estimado extrapolando los datos del 2017 (que se corresponden con las cantidades devengadas en los meses de agosto y septiembre), pero únicamente nos ha sido posible hacerlo para CEDRO, puesto que a día de hoy (principios de agosto de 2018), la SGAE no ha publicado aún su memoria anual del ejercicio 2017.

\section{Ventajas y desventajas del canon digital, y posibles sistemas alternativos}

\subsection{Ventajas y desventajas del canon}

Coincidimos con el profesor Fernando Carbajo (2016, p. 14), que defiende que un sistema de canon sobre los soportes y equipos es el más racional y eficiente, por ser el que mejor se adecúa a la esencia de la copia privada, además de que es el utilizado en casi todos los Estados europeos, opinión que comparten también las sociedades de gestión de derechos.

Adicionalmente, como hemos podido comprobar, los creadores reciben mayores cantidades con los sistemas basados en canon sobre equipos y soportes, lo que se traduce en un incentivo a la creación cultural, beneficiando a la sociedad en su conjunto.

No obstante, no podemos obviar que el regreso del canon ha sido duramente criticado por las asociaciones de usuarios, al considerar que se traduce en un importante aumento de precio de los productos afectados, que finalmente pagan los usuarios (3), y que fomentará la corrupción en las sociedades de gestión de derechos, como consecuencia de las grandes cantidades de dinero que volverán a recaudar, algo que ya ocurrió con el antiguo canon digital.

También debemos tener en cuenta que, aunque se argumenta que con el canon son las empresas tecnológicas, beneficiadas con la venta de los productos, las que pagan la compensación (Calvo, 2017, p. 19), esto no es cierto, pues estas empresas trasladarán tarde o temprano dichos costes a los usuarios finales, aun cuando los productos tengan usos distintos al de la copia privada, de forma que el canon se traduce en un incremento del precio de los equipos y soportes.

Por otra parte, la regulación actual de la compensación por copia privada en la UE plantea serios problemas relativos al mercado único, debido al diferente tratamiento que de la misma se lleva a cabo en los distintos Estados miembros (Carbajo, 2016, p. 15).

\subsection{Posibles sistemas alternativos}

Como alternativa a los dos sistemas que hemos visto (canon digital y con cargo a los PGE), hay quien aboga por eliminar totalmente el límite de copia privada, al considerar que solo sirve para enriquecer a las entidades de gestión de derechos a costa de los usuarios, que son los que finalmente pagan, y que solo una ínfima parte de lo recaudado llega a los autores (Carrasco, 2016, p. 5), pero no creemos que ésta sea la solución adecuada, ya que la eliminación de este límite incrementaría considerablemente las controversias judiciales, además de limitar el acceso a la cultura.

Por otra parte, las asociaciones de usuarios defienden que sería más adecuado implantar un canon directo sobre las propias obras protegidas, sistema ya adoptado en su día por Holanda (Calvo, 2017, p. 2), de forma que sean los titulares de los derechos los que reciban directamente la compensación, evitando así la intermediación de las sociedades de gestión (Sánchez, 2017).

No obstante, creemos que un canon de este tipo se traduciría en un desincentivo a la creación, pues el aumento del precio de las obras provoca una reducción de la demanda, que tarde o temprano hará que también se reduzca la oferta, por lo que tampoco podemos defender esta propuesta de las asociaciones de usuarios.

\section{Resumen y conclusiones}

La copia privada es un límite al derecho de reproducción, cuya compensación equitativa ha sufrido diversos cambios en España: el canon digital implantado en 2006 se sustituyó en 2011 por una compensación con cargo a los PGE, y nuevamente en 2017 se regresó al sistema basado en canon sobre soportes y equipos.

Estos cambios legislativos han afectado a todos los actores que intervienen en la creación y distribución de las obras (autores, editores, productores, intérpretes, ejecutantes, etc.), pues las cantidades que éstos perciben se ven profundamente afectadas por el sistema de compensación vigente. 
Pero los cambios también han afectado a los usuarios, no solo porque son los que finalmente pagan la compensación de una u otra forma (vía PGE o vía canon), sino porque además, la regulación del límite de copia privada tiene consecuencias importantes sobre su derecho de acceso a la cultura.

Por ello, el nuevo canon digital ha sido valorado muy positivamente por las entidades de gestión de derechos, que esperan que la recaudación recupere los niveles anteriores al año 2012, pero ha provocado importantes críticas de las asociaciones de usuarios, que consideran que se traduce en un importante incremento del precio de los equipos y soportes utilizados para las copias, que finalmente pagarán los usuarios.

Aunque defendemos el sistema de canon actual, al considerar que es claramente mejor que las alternativas existentes (PGE, eliminar el límite, y canon sobre obras), creemos que debería armonizarse en toda la UE, para evitar distorsiones del mercado interior, siendo preciso además moderar las cantidades asignadas a los equipos y soportes, al objeto de no provocar pérdida de competitividad con terceros países y reducir a su vez el coste para los usuarios.

Además, de lege ferenda, creemos que es necesario revisar y actualizar el alcance del límite de copia privada en España, para dar cobertura a los discos virtuales en Cloud Computing (ya contemplados en algunos países europeos), y también para eliminar el requisito de que la copia sea realizada $\sin$ la asistencia de terceros (Carbajo, 2016 , p. 17), algo que desde nuestro punto de vista no tiene sentido.

Por otra parte, y para evitar la reaparición de casos de corrupción en el seno de las sociedades de gestión de derechos, algo que ya ocurrió con el antiguo canon digital, es preciso aumentar los mecanismos de control sobre dichas sociedades, y exigirles una total transparencia en su gestión, especialmente en lo que respecta a la asignación y reparto de las cantidades recaudadas por copia privada, así como también sobre el destino del porcentaje de dicha recaudación que, por ley, deben dedicar a formación, promoción y fines asistenciales de sus socios (art. $155 \mathrm{TRLPI}$ ).

Finalmente, creemos que la vuelta al canon digital sobre equipos y aparatos contribuirá a recuperar el justo equilibrio entre los derechos de los autores y los de los usuarios, pues permitirá a estos últimos un mejor acceso a la cultura utilizando el límite de copia privada, y a los primeros percibir una adecuada compensación equitativa, que sin lugar a dudas incentivará la creación de nuevas obras artísticas y literarias, enriqueciendo nuestro acervo cultural.

\section{Notas}

(1) Especialmente con la digitalización, pues la calidad de las copias digitales no se degrada, haciendo posible realizar copias de las copia indefinidamente, manteniendo la calidad.

(2) Por ejemplo, el $85 \%$ de la recaudación de la SGAE en 2015 es de un acuerdo con Microsoft (Nokia), por atrasos de 2009 a 2011.

(3) Un disco duro de 1TB, que cuesta actualmente unos 40 $€+$ IVA, tiene un canon de 6,45€ + IVA (si se adquiere de forma separada), lo que supone un incremento de más del $16 \%$.

\section{Referencias}

Abbud, José Carlos; Fade, Bárbara (2010). Las leyes de derecho de autor y su relación con la preservación digital. // Ibersid. 4 (2010) 159-164. ISSN 1888-0967. http://www. ibersid.eu/ojs/index.php/ibersid/article/view/3822 (20182-27).

Aparicio, Juan Pablo (2016). Propiedad intelectual y suministro de contenidos digitales. // InDret. 3 (Julio 2016). 16 ISSN: 1698-739X. http://www.indret.com/pdf/1246.pdf (2018-2-21).

BOE (1987). Ley 22/1987, de 11 de noviembre, de Propiedad Intelectual. // BOE. 275 (17/11/1987) 34163-34176.

BOE (1996). TRLPI. Real Decreto Legislativo 1/1996, de 12 de abril, por el que se aprueba el texto refundido de la Ley de Propiedad Intelectual, regularizando, aclarando y armonizando las disposiciones legales vigentes sobre la materia. // BOE. 97 (22/4/1996) 14369-14396.

BOE (2006). Ley 23/2006, de 7 de julio, por la que se modifica el TRLPI. // BOE. 162 (8/7/2006) 25561-25572.

BOE (2008). Orden PRE/1743/2008, de 18 de junio, por la que se establece la relación de equipos, aparatos y soportes materiales sujetos al pago de la compensación equitativa por copia privada, las cantidades aplicables a cada uno de ellos y la distribución entre las diferentes modalidades de reproducción. // BOE. 148 (19/06/2008) 27842-27844.

BOE (2011). Real Decreto-ley 20/2011, de 30 de diciembre, de medidas urgentes en materia presupuestaria, tributaria y financiera para la corrección del déficit público. // BOE. 3156 (31/12/2011) 146574-146648.

BOE (2012). Real Decreto 1657/2012, de 7 de diciembre, por el que se regula el procedimiento de pago de la compensación equitativa por copia privada con cargo a los PGE. // BOE. 295 (8/12/ 2012), 84141-84148.

BOE (2017). Real Decreto-ley 12/2017, de 3 de julio, por el que se modifica el TRLPI, en cuanto al sistema de compensación equitativa por copia privada. // BOE. 158 (4/7/2017) 56444-56454.

Calvo, Juan (2017). El futuro del canon digital en España tras las Sentencias del TJUE de 9 de junio de 2016 y del TS de 10 de noviembre de 2016. // Revista Aranzadi Doctrinal. 2 (2017) 1-19 (online). ISSN: 1889-4380.

Carbajo, Fernando (2002). Publicaciones electrónicas y propiedad intelectual. // Editorial Colex, Madrid. 2002.

Carbajo, Fernando (2010). Creación, edición y lectura en la sociedad de la información: entre la propiedad intelectual y el acceso a la cultura. // Pliegos de Yuste $\mathrm{n}^{0}$ 11-12 (2010) 127-134. ISSN: 1697-0512. http://www.pliegosdeyuste.eu/n1112pliegos/pdfs/125-134.pdf (2018-2-16).

Carbajo, Fernando (2016). El retorno del canon digital. Consecuencias previsibles de la STJUE de 9 de junio de 2016. // Ars luris Salmanticensis Vol. 4-2 (2016) 11-20. 
ISSN-e 2340-5155. http://revistas.usal.es/index.php/ais/ article/view/15254/15836 (2018-2-20).

Carrasco, Ángel (2016). A las barricadas, que vuelve el canon digital. // Actualidad jurídica Aranzadi, n 921 (2016) 5. ISSN 1132-0257.

CEDRO (2018). Memorias anuales. http://www.cedro.org/cedro/memorias (2018-1-28).

DOUE (2001). DDA. Directiva 2001/29/CE del Parlamento Europeo y del Consejo, del Parlamento Europeo y del Consejo, de 22 de mayo de 2001, relativa a la armonización de determinados aspectos de los derechos de autor y derechos afines a los derechos de autor en la sociedad de la información. // Diario Oficial de la UE (DOUE). 167 (22/6/2001) 10-19.

García, Jesús Félix (2017). El nuevo canon digital en España // Revista quincena fiscal $n^{\circ} 4$ (2017), 1-20 (online). ISSN 1132-8576.

González, José Luis (2016). Libro electrónico e interoperabilidad. Formatos y medidas tecnológicas de protección. // TELOS. 104 (2016), 69-77. ISSN 0213-084X. http://telos.fundaciontelefonica.com (2018-2-22).

González, José Luis (2017). Régimen jurídico de las obras huérfanas en España. // Ibersid Vol. 11-2 (2017) 35-40 ISSN 1888-0967. http://www.ibersid.eu/ojs/index.php/ ibersid/article/view/4417. (2018-2-16).

Riera, Patricia (2001). Derechos de autor y acceso a la información: los conflictos en la era digital // Anuari de Biblioteconomia, Documentació i Informació: Bibliodoc 2001/
2002 113-122. ISSN 1885-0685, http://www raco.cat/index.php/Bibliodoc/article/view/16640/16482 (2018-2-16).

Sánchez, Luis Javier (2017). ¿Quién pagará el "nuevo" canon digital? // Asociación de internautas. https://www.internautas.org/sincanon/html/9555.html (2018-2-25)

SGAE (2018). Memorias anuales. http://www.sgae.es/esEs/SitePages/corp-gobierno-corporativo.aspx (2018-128)

TJUE (2010). Sentencia del TJUE de 21/10/2010, asunto PADAWAN, C 467-08.

TJUE (2015). Sentencia del TJUE de 5/3/2015, asunto COPYDAN, C 463-12.

TJUE (2016). Sentencia del TJUE de 9/6/2016, asunto EGEDA, C 470-14.

VUD (2017). Ventanilla Única Digital, informe Ejecutivo Ejercicio 2017. https://www.mecd.gob.es/cultura-mecd/areas -cultura/propiedadintelectual/limite-legal-copia-privada/ gestion.html (2018-8-24).

Wijminga, Hester et al. (2017). International Survey on Private Copying - Law and Practice 2016. // Publicación de la OMPI, 2017. (2018-1-27). http://www.wipo.int/publications/es/details.jsp?id=4183.

Enviado: 2018-03-06. Segunda versión: 2018-08-02 Aceptado: 2018-09-05. 